\title{
Developing a Framework for Scoping Digital Twins in the Process Manufacturing Industry
}

\author{
Matteo PERNO ${ }^{\mathrm{a}, 1}$ and Lars HVAM ${ }^{\mathrm{a}}$ \\ ${ }^{a}$ Department of Technology, Management and Economics, Technical university of \\ Denmark, Kongens Lyngby, Denmark
}

\begin{abstract}
The process of scoping Digital Twin projects can prove to be daunting for process manufacturing companies, given the novelty of the concept and the broad range of technologies upon which it is built. The current literature lacks research on the process of scoping Digital Twins in a process manufacturing context. The present paper addresses this gap by introducing a framework for scoping Digital Twins in the process manufacturing industry. The framework is based on the existing literature and on the experience gained at an international process manufacturing company. The proposed framework is designed to address the key challenges that companies in the process manufacturing industry need to face when scoping Digital Twin projects. Therefore, the framework is structured into four points: (1) Identification of key stakeholders and their requirements, (2) Building blocks definition, (3) Selection of asset to twin, and (4) Use cases development. By addressing these points, the time and resources required to develop a Digital Twin can be significantly reduced.
\end{abstract}

Keywords. Digital Twin, Industry 4.0, Scoping, Framework.

\section{Introduction}

The latest developments in IT include techniques such as Big Data analytics, Machine Learning, Industrial Internet of Things (IIoT) and Cloud Computing [1][2]. These technologies represent a valuable opportunity for process manufacturing companies of any size and industry type to digitalize their production processes and make the most out of their data [1][3][4].

The Digital Twin is a concept that encompasses all of the above-mentioned techniques. From a broad perspective, a Digital Twin can be defined as a virtual representation of an existing physical system [5]. A Digital Twin leverages real-time as well as historic data to obtain valuable insights on the status and performance of an asset [4]. The current literature on Digital Twins is lacking research on the process of scoping them. Thus, this paper aims at filling this gap in the literature by introducing a framework designed to address the challenge of scoping Digital Twin projects. The proposed framework focuses on the process manufacturing industry, and the possibility to extend it to other industries will be argued later on in this paper. The framework has been developed, applied and validated in an industrial context. The case company chosen for

\footnotetext{
${ }^{1}$ Corresponding Author. matper@dtu.dk
} 
this project is Haldor Topsøe, an international engineering company based in Denmark, leader in the production of catalysts for heterogeneous catalysis. The products manufactured by Haldor Topsøe are chemical substances produced using continuous flow production lines. The products produced by the case company are used in a variety of applications to speed up specific chemical reactions by lowering the activation energy required for the reaction to take place. In heterogeneous catalysis, the catalyst is in a different phase than the reactants, i.e. the catalyst is solid and the reactants are liquid or gas. Haldor Topsøe has been selected as the case company for this study as it is currently going through the process of scoping a Digital Twin project for one of their production lines. Furthermore, the researchers are actively involved in several research projects within the company. In particular, one of the authors is currently working on a $\mathrm{PhD}$ project in collaboration with Haldor Topsøe, hence the researchers have direct access to the company's knowledge and data.

Given the novelty of Digital Twins, the case company expressed the need for a structured approach to scope their Digital Twin project. The framework proposed in this study is based on current literature on Digital Twins, as well as on the experience gained at the case company. The rest of this paper is structured as follows: Section 1 describes the methodology used to conduct this study. Section 2 presents the findings from the literature review. Section 3 outlines the proposed framework. Section 4 discusses the application of the framework at the case company. Section 5 elaborates on the benefits obtained from the framework application. Finally, section 6 concludes the paper and proposes suggestions for further research.

\section{Methodology}

The methodology applied to this study draws directly from Design Science Research (DSR), as described by Vaishnavi and Kuechler [6]. The research process started by building awareness on the problem through a literature study. Then, an artifact was developed in the form of a framework and applied at Haldor Topsøe. The evaluation of the framework was based on meetings with the key stakeholders of the Digital Twin project at the case company. Hence, the methodology used in this study has been summarized into three parts: (1) Literature review, (2) Framework development, and (3) Framework application. In the following subsections, each part is outlined.

\subsection{Literature Review}

The literature review performed in this study fulfilled two purposes: first, to gather the necessary knowledge in order to identify the steps to include in the proposed framework. Secondly, to identify the current gap in the literature and therefore assess the potential of this study to contribute to the research on Digital Twins.

The literature review is divided into two parts: (1) Studying the latest research on Digital Twins and (2) Searching for valuable inputs on how to scope Digital Twins in industrial applications.

\subsection{Framework Development}

The framework introduced in this paper was developed based upon the literature review and the work done at the case company Haldor Topsøe A/S. The framework was setup 
in close collaboration between industry practitioners and researchers with a background in Industry 4.0, Smart Manufacturing, and Supply Chain Management. The results of the literature review process have been categorized into four groups, given by elements that were discussed in almost all of the reviewed papers. These groups have been discussed with the case company and used as a basis for the development of the framework.

\subsection{Framework Application}

After deciding upon which steps to include in the framework, the researchers applied each step of the framework to the case company. The results have been discussed in a meeting between the researchers and the key stakeholders of the Digital Twin project at the case company.

\section{Literature Review}

This section describes the literature review performed for this study, which can be divided into two parts. In the first part, the current literature on Digital Twins is reviewed, with the purpose of getting an overall understanding of the concept of Digital Twins and its origin. Then, inputs on how to scope Digital Twin projects are gathered and outlined. These will be used for the development of the framework. The Scopus and Web of Science Core Collection databases have been used for the literature search.

\subsection{Digital Twins Overview}

Although the most commonly used terminology to define a digital representation of a physical asset is Digital Twin, this concept can be found under other names. In the literature, the most common synonym for Digital Twins is Digital Shadows [7]. In industry, Microsoft refers to Digital Twins as Device Twins [8], while Amazon uses the term Device Shadows [9]. Since the vast majority of the literature uses the name Digital Twin, for the sake of convenience and to avoid confusion on the terminology, the name Digital Twin will be used for the rest of the study.

The first study proposing the idea of a digital representation of an existing asset dates back to 1994 from the healthcare sector [10]. However, the Digital Twin developed in this case consists in a static representation, with no connection between the physical entity and its digital counterpart. The idea of linking the real and virtual spaces through a real-time and continuous flow of data was first introduced by Professor Michael Grieves at the University of Michigan in 2002 [11]. The name Digital Twin was introduced for the first time in 2011 by Tuegel et al. [12].

However, Digital Twins gained worldwide attention only in 2016, after the research and advisory firm Gartner listed them among the top 10 strategic technology trends for 2017 [13]. This event marked the beginning of an exponential growth in the number of publications on Digital Twins. Although the research on this topic is growing exponentially, it has not reached a mature stage yet. A symptom of this is the fact that the definition of a Digital Twin is still subject to debate, both from industry practitioners and reserachers. This is illustrated in table 1 below, which presents an overview of the most significative definitions of a Digital Twin found in the literature in chronological order, from the first formulation in 2011 to the most recent. The search string used to search for literature in this case is ("Digital Twin" OR "Digital Shadow"). Only 
publications from 2011 and later have been considered and sorted in terms of number of citations. Among the top resulting publications, only those proposing an original definition of the concept of Digital Twin have been considered:

Table 1. Overview of the definitions of a Digital Twin found in the literature.

\begin{tabular}{|c|c|c|c|}
\hline Publication & Year & Definition & $\begin{array}{l}\text { Citations } \\
\text { (Scopus) }\end{array}$ \\
\hline $\begin{array}{l}\text { Tuegel et al. } \\
\qquad 14]\end{array}$ & 2011 & $\begin{array}{c}\text { "An ultrahigh fidelity model of an individual aircraft by tail number } \\
\text { that serves as a reengineering of structural life prediction and } \\
\text { management." }\end{array}$ & 162 \\
\hline $\begin{array}{l}\text { Glaessgen } \\
\text { and Stargel } \\
\quad[15]\end{array}$ & 2012 & $\begin{array}{c}\text { "An integrated multiphysics, multiscale, probabilistic simulation of } \\
\text { an as-built vehicle or system that uses the best available physical } \\
\text { models, sensor updates, fleet history, etc., to mirror the life of its } \\
\text { corresponding flying twin." }\end{array}$ & 113 \\
\hline $\begin{array}{l}\text { Rosen et al. } \\
\quad[16]\end{array}$ & 2015 & $\begin{array}{l}\text { "Realistic model of the current state of the process and their own } \\
\text { behavior in interaction with their environment in the real world." }\end{array}$ & 227 \\
\hline $\begin{array}{l}\text { Grieves and } \\
\text { Vickers [11] }\end{array}$ & 2017 & $\begin{array}{l}\text { "A set of virtual information constructs that fully describes a } \\
\text { potential or actual physical manufactured product from the micro } \\
\text { atomic level to the macro geometrical level." }\end{array}$ & 103 \\
\hline $\begin{array}{l}\text { Lu et al. [17] } \\
\text { adapted from } \\
\text { Schleich et } \\
\text { al. [18] }\end{array}$ & 2019 & $\begin{array}{l}\text { "A high-fidelity representation of the operational dynamics of its } \\
\text { physical counterpart, enabled by near real-time synchronization } \\
\text { between the cyberspace and physical space." }\end{array}$ & 134 \\
\hline
\end{tabular}

As table 1 above shows, multiple different viewpoints on the concept of Digital Twin can be found in the literature. For the purpose of this study, Digital Twins have been considered as high-fidelity representations of the operational status of their corresponding physical twin, enabled by near real-time synchronization and data exchange between the digital and physical twins (adapted from Lu et al. [17]).

\subsection{Scoping Digital Twins}

The second part of the literature review process focused on searching for literature on the process of scoping Digital Twin projects. In the Scopus and Web of Science Core Collection databases, the following search string was used: ("Digital Twin" OR "Digital Shadow") AND ("scope" OR "scoping" OR "framework" OR "frame" or "framing"). The resulting literature indicated a lack of research specifically addressing the scoping process in Digital Twin projects. However, most of the publications reviewed throughout the whole literature review process indicated a pattern in terms of the choices made by the authors in order to narrow down the focus of their studies on Digital Twins. Such recurring elements have been categorized into four groups, which are presented in table 2 below. 
Table 2. Part of the reviewed literature on Digital Twins, in relation to each of the identified groups. Each $X$ marks an association between a publication and one of the identified groups.

\begin{tabular}{ccccc}
\hline Publication & $\begin{array}{c}\text { Group 1 } \\
\text { Stakeholders } \\
\text { management }\end{array}$ & $\begin{array}{c}\text { Group 2 } \\
\text { Features and } \\
\text { functionalities }\end{array}$ & $\begin{array}{c}\text { Group 3 } \\
\text { Physical asset to } \\
\text { be twinned }\end{array}$ & $\begin{array}{c}\text { Group 4 } \\
\text { Application areas } \\
\text { and use cases }\end{array}$ \\
\hline Macchi et al. [19] & & & $\mathrm{X}$ & $\mathrm{X}$ \\
Rosen et al. [16] & $\mathrm{X}$ & & $\mathrm{X}$ & $\mathrm{X}$ \\
Lu et al. [17] & $\mathrm{X}$ & $\mathrm{X}$ & $\mathrm{X}$ & $\mathrm{X}$ \\
Durão et al. [20] & $\mathrm{X}$ & $\mathrm{X}$ & $\mathrm{X}$ \\
Josifovska et al. [21] & & $\mathrm{X}$ & & $\mathrm{X}$ \\
Ciavotta et al. [22] & $\mathrm{X}$ & $\mathrm{X}$ & & $\mathrm{X}$ \\
Rabah et al. [23] & $\mathrm{X}$ & $\mathrm{X}$ & & $\mathrm{X}$ \\
Barricelli et al. [24] & & & & \\
\hline
\end{tabular}

\section{Framework Development}

The results of the literature study and the four groups from table 2 have been discussed during a meeting at the case company. As a result, the four groups identified in table 2 have been adapted and revised to fit the needs and characteristics of the case company.

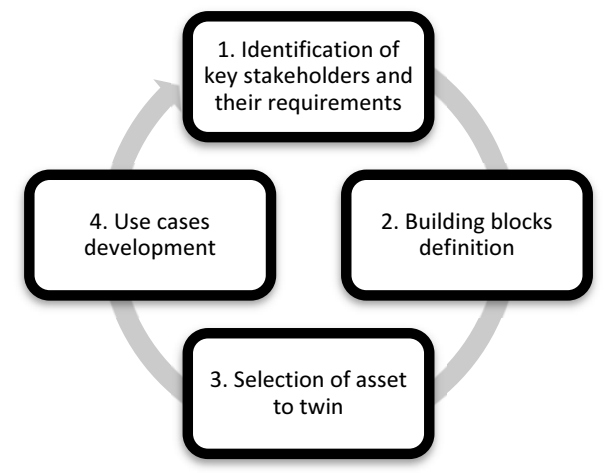

Figure 1. Framework for scoping Digital Twin projects.

Each of the four steps is outlined in the following subsections.

\subsection{Identification of key stakeholders and their requirements}

The first step in a Digital Twin project is to identify the stakeholders of the project and their requirements. The tasks to carry out in this step are: (1) Listing the stakeholders of the Digital Twin project, (2) Dividing the stakeholders into categories, (3) Gathering and prioritising stakeholder requirements, and (4) Building a project team.

The first task consists in creating a comprehensive list of all people who are going to be involved in the Digital Twin project. This list should include both internal employees at the company and external stakeholders. Examples of possible internal stakeholders are: operators on the production line, process engineers, maintenance staff, product managers, product developers or software developers. External stakeholders can be i.e. suppliers, customers, investors, or external partners. Each of these stakeholders may have different requirements and needs when it comes to the Digital Twin, and all of 
them need to be taken into consideration during the scoping process. To create a list of all stakeholders, different techniques can be used i.e. brainstorming sessions and unstructured interviews [25].

Once the full list is ready, the second task consists in splitting the identified stakeholders into categories, for example by job title, department, division, or market.

In the third task, the requirements of each of the stakeholders previously identified are gathered and prioritised. For the requirements gathering, tools from the Rational Unified Process (RUP) such as process-flow charts and use-case diagrams can be used [26]. Alternatively, requirements can be identified through meetings, interviews, or surveys with the selected stakeholders. To prioritise the requirements, the MoSCoW (Must have, Should have, Could have, Want to have but not this time around) prioritization technique can be used [27].

Finally, in the fourth task the Digital Twin project team is built. For this task, Hong et al. [28] suggest that successful IT projects possess four key factors: (1) The involvement of management executives, (2) the involvement of project managers with IT skills and leadership, (3) having skilled and experienced project team members, and (4) project control and reporting practices. Ideally, a project team should consist at least in a Project Owner, a Project Manager and a Digital Twin Specialist, who is a professional figure with knowledge and expertise in Digital Twins and their implementation. The role of the Digital Twin specialist is to provide the technical expertise to support the Project Manager and the Project Owner throughout the whole project. The output of this first macro-step is a clear and well defined list of stakeholders, together with their requirements and needs. Furthermore, the project ownership and governance are outlined.

\subsection{Building blocks definition}

In a manufacturing context, a Digital Twin can be used for a variety of purposes; for example, it can be used for simulation, monitoring, controlling, running diagnostics, and making predictions [18]. Lu et al. [17] identified the following building blocks for a Digital Twin: (1) The information model, which is the standard used to abstract the physical asset in the digital world, (2) the communication network, including the standard communication protocol used for the real-time data exchange between the physical asset and its Digital Twin, and (3) Big Data processing, or the ability of the Digital Twin to process large volumes of data through statistical analysis and predictive models to generate valuable insights on the status of the production process.

The building blocks of the Digital Twin are a consequence of the stakeholders requirements and the particular problem the company wants to solve through this technology. This means that if a company wants to i.e. implement a predictive maintenance strategy to minimize downtime and perform maintenance operations only when necessary, Machine Learning will be an essential building block for the final Digital Twin system. The purpose of the building blocks selection step is to clarify the features and functionalities of the digital twin to all of the project's stakeholders.

\subsection{Selection of asset to twin}

Another important decision to make when scoping Digital Twin projects regards the choice of which physical asset needs to be twinned in the digital world. In a manufacturing context, any asset can be twinned: a single product, an asset on the 
production line, the whole production line, or even the whole company, as long as large amounts of relevant data are available to represent the asset in the digital world.

$\mathrm{Lu}$ et al. [17] focus on the product level, describing how Digital Twins can be beneficial in product manufacturing, product design and product service. This is already happening in the automotive industry, where car manufacturers are creating Digital Twins of each car they produce. The sensors embedded in the cars continuously send performance data back to the manufacturer, including data on breakdowns or failures of the car's components. This allows the car manufacturer to improve their production process and produce better and more reliable products. Josifovska et al. [21] discuss the development of Digitals Twins for machines and components on a production line within a cyber-physical system. Macchi et al. [19] take a more holistic approach, considering Digital Twins of entire production systems.

\subsection{Use cases development}

A variety of different use cases can be developed for a Digital Twin. Macchi et al. [29] identified five industrial use cases for Digital Twins within the context of Asset Management (AM): (1) Asset configuration, (2) Asset reconfiguration, (3) Asset reconfiguration and planning, (4) Asset commissioning, and (5) Asset condition monitoring and health assessment. Rabah et al. [23] focus on the synergy between Digital Twins and Augmented Reality (AR), which can be used as a tool to improve the interaction between users and the Digital Twin. They argue that this can significantly improve the predictive maintenance capabilities of the company. Rosen et al. [16] discuss how a Digital Twin can be used: (1) To plan offers and orders based on statistical assumptions using real-time data, and (2) To execute operations automatically through simulation models of the capabilities of production units, product handover, as well as visualization of process data and asset management.

Developing use cases for a Digital Twin can help the stakeholders getting a clear understanding of how the Digital Twin can be used and what benefits it can bring in realworld situations.

\section{Framework Application}

Each step of the presented framework has been applied to the case company Haldor Topsøe A/S. The following subsections discuss the outcome of the application of each step to the case company.

\subsection{Identification of key stakeholders and their requirements}

First of all, the key stakeholders for the Digital Twin project at the case company have been identified. Initially, the researchers had a brainstorming session to develop a list of all potential stakeholders [25]. The resulting list has been discussed and subsequently approved and validated through unstructured interviews with the managers at the case company responsible for initiating the Digital Twin project. During a meeting with the stakeholders of the Digital Twin project at the case company, it was decided to categorize the identified stakeholders by department and job title, in order to get a clear understanding of the resources involved in the Digital Twin project. The results of the categorization process are shown in figure 2 below. 
To identify the requirements of each stakeholder, different methods have been used, depending on factors such as the role and job function, the availability, and the geographical location of the stakeholder. In some cases, stakeholders were asked to prepare a document describing their requirements for the Digital Twin. In other cases, unstructured interviews have been conducted, either in person or through phone calls. The final list of requirements has then been verified and discussed in a meeting with the stakeholders at the case company, where the MoSCoW technique was used to prioritize the requirements. After merging duplicate requirements expressed by multiple stakeholders, a total of ten requirements have been identified. Of these, four have been classified as Must have, four as Should have, one as Could have and one as Would have. It is important to underline that the list of requirements and their classification might be subject to change over the course of the Digital Twin project. Finally, a Digital Twin project team was built. This consists in a Project Owner, a Project Manager, and a Digital Twin Specialist.

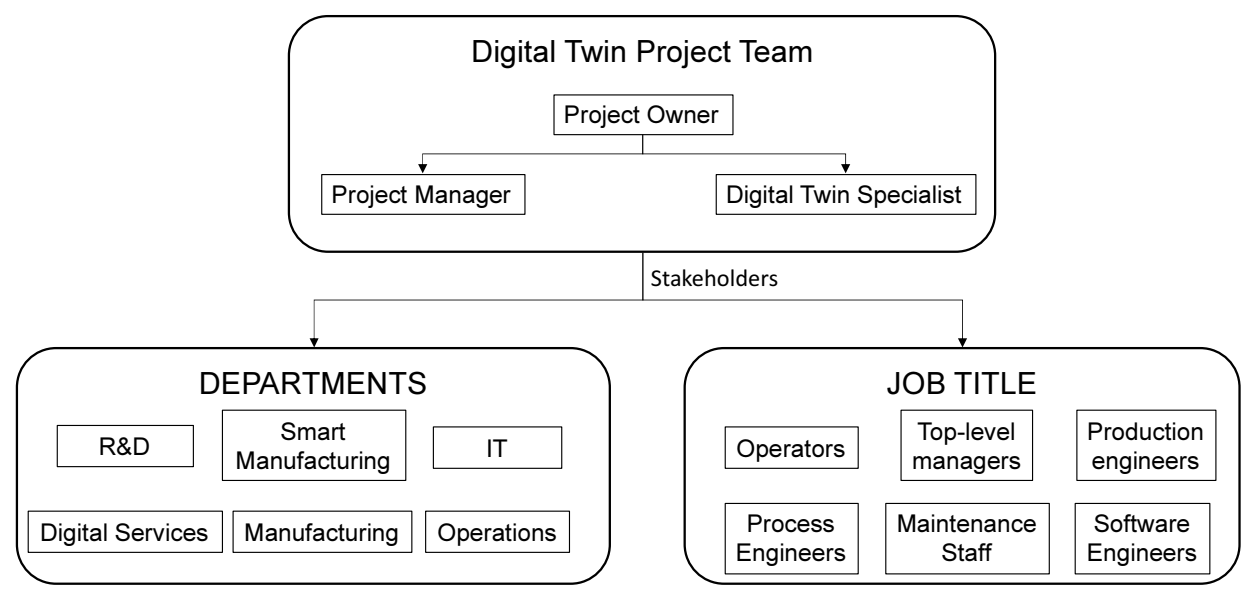

Figure 2. Project team and stakeholders at the case company

\subsection{Building blocks definition}

As a result of several meetings between the researchers and the key stakeholders at the case company, the following building blocks have been identified for the Digital Twin:

- Real-time data acquisition: The Digital Twin must be able to receive large amounts of data in real-time that can be used to generate insights on the current and future status of the system being twinned.

- Artificial Intelligence/Machine Learning models: Machine Learning models are going to be generated and trained to predict i.e. when a piece of equipment is going to break down, or the quality of a finished product at the end of the production process.

- Virtual Reality: Full-scale 3D models of the asset to be twinned are going to be created and made accessible to anyone at the case company. These can be used for different use-cases i.e. remote assistance or training.

- Simulation software: The Digital Twin will include simulation software that is suitable for modelling the production process at the case company. The 
simulation models built with the chosen simulation software will be fed realtime and historic data to test different case scenarios.

- Notification system: A notification system will be implemented, in order to notify the operators working on the production line that an error or malfunction is going to occur on the production line, in a timely manner. This feature enables predictive maintenance, as operators can proactively intervene on the malfunction, avoiding costly downtime.

- Data visualization software: The possibility to visualize data coming in realtime from the sensors on the production line in a graphical format was considered to be crucial for the Digital Twin. This feature allows the end users to easily visualize the trend of the production parameters and compare them to the target value as well as the upper and lower boundaries.

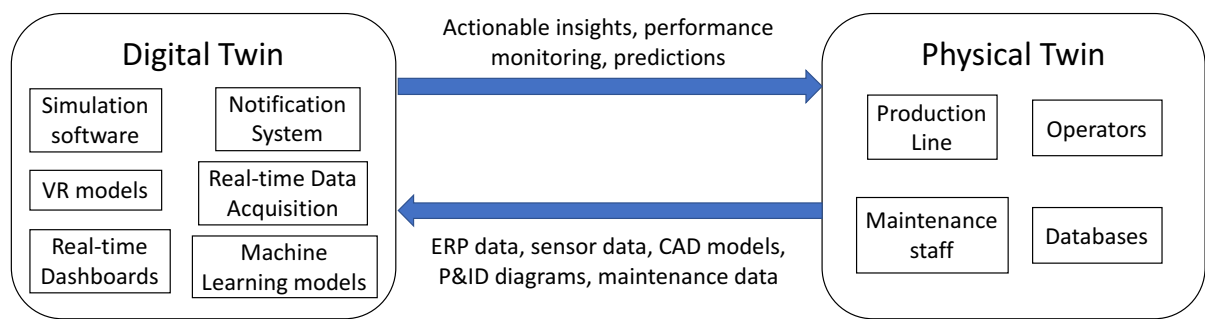

Figure 3. Digital Twin building blocks at the case company

The building blocks discussed above (see also Figure 3) have been chosen according to the needs and expectations of the stakeholders at the case company, in regard to the digital twin project. These building blocks have been represented and summarized on a dashboard that has been validated at the case company and will be used as a reference model for the features and functionalities of the Digital Twin in development.

\subsection{Selection of asset to twin}

At the case company, the end goal for the Digital Twin project is to model a relatively small and simple production line. To reach this goal, it was decided to start by creating a first prototype of a Digital Twin for a single machine on the production line.

The prototype will initially have a number of basic features, and an iterative process will be followed for its development, where new features will be implemented at each iteration, and feedback will be gathered from the end users of the system. Once the first prototype is ready, the same approach will be used for another machine on the same production line, and the two Digital Twin prototypes will be connected to each other by exchanging data. This will generate the first Digital Twin Aggregate (DTA), which is an aggregate of several different Digital Twins [11]. This process will be repeated for each machine on the production line, until a DTA of the whole line will be created.

\subsection{Use cases development}

At the case company, six use cases have been identified for Digital Twin technology: 
- Troubleshooting: By feeding real-time sensor data to a Machine Learning model, Digital Twins can predict equipment breakdown before it occurs, so that maintenance activities can be scheduled only when they are needed, avoiding both costly process downtime and unnecessary maintenance activities. Also, Machine Learning models can detect anomalies in sensor readings and alert the operators on the production line so that errors can be quickly addressed and resolved.

- Training: New employees can be trained in a Virtual Reality environment on how to use the equipment they need for their daily work. In this case, tutorials can be designed by developers and used by new employees to learn and practice by interacting with digital representations of the equipment. This avoids the risk of costly mistakes on real equipment, and significantly reduces the cost of training new employees.

- Remote assistance: If part of the company's production was to be outsourced from another country, the employees in that country could ask for assistance to an experienced engineer at the company's headquarters. In this situation, both parties can access the same VR model in real-time and look and interact with the same piece of equipment, making the assistance process much simpler and clearer.

- Simulation: A Digital Twin constantly updates itself with new data coming from sensors on the physical asset. These data are used to run simulations to test different case scenarios and answer different questions. For example, what would happen if the speed of a machine on the production line is increased or decreased by a certain percentage? What would happen to the rest of the line? Would a bottleneck be generated? These insights can be used to optimize the production process. Furthermore, simulation can be used to test the production of new products in a digital environment, avoiding the need to run test productions on the real production line.

- Maintenance: The Digital Twin can help predicting when maintenance operations need to be carried out to avoid equipment breakdown, as well as predicting the quality of the output coming out of the line. When the Digital Twin makes a prediction, the output should be a detailed warning message alerting the operators on the production line.

- Operations: From the Digital Twin platform, the end users will be able to access all available information about the twinned asset. This includes P\&ID diagrams, availability in stock, specifications, manuals etc. This means for example that in a real-case scenario the Digital Twin can send an alert when a spare part is not available in stock and an order can be placed for that part directly from within the Digital Twin platform.

The use cases listed above have been validated at the case company during a meeting with the stakeholders of the Digital Twin project.

\section{Results}

The presented framework for scoping Digital Twins in process manufacturing companies has been applied to the case company Haldor Topsøe A/S, a world leading manufacturer of catalysts for heterogeneous catalysis. The application of the proposed framework 
helped the Digital Twin project team at the case company getting an early clarification of the scope of the Digital Twin to be developed. This has proven to be very valuable, as it enabled the alignment of expectations and requirements of the project's stakeholders. Furthermore, the company benefited from having a clear overview of the key people responsible for the Digital Twin project, what the twin is going to look like, and how it is going to benefit the whole organization. The benefits given by the application of the framework have been validated through a meeting with the stakeholders at the case company. Therefore, the defined scope represents a solid basis for the following steps of the Digital Twin project at Haldor Topsøe. The next step for the project team will be to develop a Minimum Viable Product (MVP) of a Digital Twin with the basic building blocks identified during the scoping process. The MVP will be presented iteratively to the end users of the system, with the purpose of gathering their feedback and user experience. This will be used to improve the MVP by adding new features or improving the existing ones at each iteration.

\section{Conclusion and further research}

This study contributes to the literature on Digital Twins by introducing a framework aimed at scoping Digital Twin projects in the process manufacturing industry. The development of the framework is based on the literature and on the experience gained from scoping a Digital Twin project at an international process manufacturing company. The four steps included in the framework are: (1) Identification of key stakeholders and their requirements, (2) Building blocks definition, (3) Definition of asset to twin, and (4) Use cases development.

The proposed framework has proven to be an effective and beneficial tool for defining the scope of the case company's Digital Twin project and reducing its complexity. The benefits gained by the case company have been evaluated during a dedicated meeting with the key stakeholders of the digital twin project at the case company. The literature study showed that the current literature on Digital Twins has a dearth of solutions when it comes to scoping Digital Twin projects. The experience gained at Haldor Topsøe A/S showed that the process of scoping a new technology such a Digital Twin can represent a challenge, if not approached systematically.

For further research, the authors are planning on extending the study by applying the proposed framework to other companies working with continuous flow production lines. Involving more companies such as Rockwool, GEA, Arla and Carlsberg and working with more case studies would further validate the framework and increase its generalizability.

\section{References}

[1] P. Augustine, The industry use cases for the Digital Twin idea, 1st ed., vol. 117, no. 1. Elsevier Inc., 2020 .

[2] J. Vatn, "Industry 4.0 and real-time synchronization of operation and maintenance," Saf. Reliab. Safe Soc. a Chang. World - Proc. 28th Int. Eur. Saf. Reliab. Conf. ESREL 2018, pp. 681-686, 2018.

[3] N. Kockmann, "Digital methods and tools for chemical equipment and plants," React. Chem. Eng., vol. 4, no. 9, pp. 1522-1529, 2019.

[4] N. Susila, A. Sruthi, and S. Usha, Impact of cloud security in digital twin, 1st ed., vol. 117, no. 1. Elsevier Inc., 2020. 
[5] W. Kritzinger, M. Karner, G. Traar, J. Henjes, and W. Sihn, "Digital Twin in manufacturing: A categorical literature review and classification,” IFAC-PapersOnLine, vol. 51, no. 11, pp. 1016-1022, 2018.

[6] V. Vaishnavi, B. Kuechler, and S. Petter, "Design Science Research in Information Systems," Ais, no. 1, pp. 1-45, 2004.

[7] G. W. G. Tseng, C. Q. G. Chen, K. Erkorkmaz, and S. Engin, "Digital shadow identification from feed drive structures for virtual process planning," CIRP J. Manuf. Sci. Technol., vol. 24, pp. 55-65, 2019.

[8] Microsoft.com, "Understand and use device twins in IoT Hub," 2019. [Online]. Available: https://docs.microsoft.com/en-us/azure/iot-hub/iot-hub-devguide-device-twins.

[9] Amazon.com, "Device Shadow Service for AWS IoT," 2019. .

[10] R. Sc and L. We, "Coronary Arteries: New Design for Three-dimentional Arterial Phantoms," 1994.

[11] M. Grieves and J. Vickers, "Digital Twin: Mitigating Unpredictable, Undesirable Emergent Behavior in Complex Systems," in Transdisciplinary Perspectives on Complex Systems: New Findings and Approaches, F.-J. Kahlen, S. Flumerfelt, and A. Alves, Eds. Cham: Springer International Publishing, 2017, pp. 85-113.

[12] M. Grieves, Virtually Perfect: Driving Innovative and Lean Products through Product Lifecycle Management. 2011.

[13] Gartner, “Gartner's Top 10 Strategic Technology Trends for 2017," Smarter With Gartner, 2016. [Online]. Available: https://www.gartner.com/smarterwithgartner/gartners-top-10-technologytrends-2017/. [Accessed: 05-Nov-2019].

[14] E. J. Tuegel, A. R. Ingraffea, T. G. Eason, and S. M. Spottswood, "Reengineering Aircraft Structural Life Prediction Using a Digital Twin,” Int. J. Aerosp. Eng., vol. 2011, no. November 2015, pp. 1$14,2011$.

[15] E. H. Glaessgen and D. S. Stargel, "The digital twin paradigm for future NASA and U.S. Air force vehicles," Collect. Tech. Pap. - AIAA/ASME/ASCE/AHS/ASC Struct. Struct. Dyn. Mater. Conf., pp. $1-14,2012$.

[16] R. Rosen, G. Von Wichert, G. Lo, and K. D. Bettenhausen, "About the importance of autonomy and digital twins for the future of manufacturing," IFAC-PapersOnLine, vol. 28, no. 3, pp. 567-572, 2015.

[17] Y. Lu, C. Liu, K. I.-K. Wang, H. Huang, and X. Xu, "Digital Twin-driven smart manufacturing: Connotation, reference model, applications and research issues," Robot. Comput. Integr. Manuf., vol. 61, no. August 2019, p. 101837, 2020.

[18] B. Schleich, N. Anwer, L. Mathieu, and S. Wartzack, "Shaping the digital twin for design and production engineering," CIRP Ann. - Manuf. Technol., vol. 66, no. 1, pp. 141-144, 2017.

[19] M. Macchi, I. Roda, E. Negri, and L. Fumagalli, "Exploring the role of Digital Twin for Asset Lifecycle Management," IFAC-PapersOnLine, vol. 51, no. 11, pp. 790-795, 2018.

[20] L. Durão, S. Haag, R. Anderl, K. Schützer, and E. Zancul, Digital twin requirements in the context of Industry 4.0. 2018.

[21] K. Josifovska, E. Yigitbas, and G. Engels, "Reference Framework for Digital Twins within CyberPhysical Systems," Proc. - 2019 IEEE/ACM 5th Int. Work. Softw. Eng. Smart Cyber-Physical Syst. SESCPS 2019, pp. 25-31, 2019.

[22] M. Ciavotta, M. Alge, S. Menato, D. Rovere, and P. Pedrazzoli, "A Microservice-based Middleware for the Digital Factory,” Procedia Manuf., vol. 11, no. June, pp. 931-938, 2017.

[23] S. Rabah et al., "Towards improving the future of manufacturing through digital twin and augmented reality technologies," Procedia Manuf., vol. 17, pp. 460-467, 2018.

[24] B. R. Barricelli, E. Casiraghi, and D. Fogli, "A survey on digital twin: Definitions, characteristics, applications, and design implications," IEEE Access, vol. 7, no. Ml, pp. 167653-167671, 2019.

[25] J. M. Bryson, "What to do when stakeholders matter: Stakeholder Identificatixon and analysis techniques," Public Manag. Rev., vol. 6, no. 1, pp. 21-53, 2004.

[26] P. Compton and R. Jansen, "Compton and Jansen 'A philosophical basis for knowledge acquisition' A philosophical basis for knowledge acquisition,” pp. 1-18, 1989.

[27] K. Bittner, Use Case Modeling. Addison-Wesley Longman Publishing Co., Inc., 2002.

[28] L. Hong, Y. Wang, J. Weng, and X. Chen, "A study of the structure of IT project team: For developing a more efficient team," 11th Int. Conf. Serv. Syst. Serv. Manag. ICSSSM 2014 Proceeding, pp. 1-5, 2014.

[29] E. Negri, L. Fumagalli, and M. Macchi, "A Review of the Roles of Digital Twin in CPS-based Production Systems,” Procedia Manuf., vol. 11, pp. 939-948, Jan. 2017. 